\title{
Article
}

Doi 10.5943/sif/5/1/6

\section{Dikaryotization seems essential for hypha formation and infection of coccid in the life-cycle of Auriculoscypha anacardiicola}

\section{Thomas $A$ and Manimohan $P$}

Department of Botany, University of Calicut, Kerala, 673 635, India

Thomas A, Manimohan P 2020 - Dikaryotization seems essential for hypha formation and infection of coccid in the life-cycle of Auriculoscypha anacardiicola. Studies in Fungi 5(1), 66-72, Doi 10.5943/sif/5/1/6

\begin{abstract}
Auriculoscypha, a monotypic basidiomycetous genus with $A$. anacardiicola as the only known species, is endemic to southwest India where it is seen in triple symbioses with a coccid and anacardiaceous trees involving interactions between three trophic levels. The present study was an attempt to verify the hypothesis that dikaryotization is a prerequisite for hypha formation and coccid-infection in the life-cycle of $A$. anacardiicola. Light-microscopic observations using Giemsa staining and fluorescent microscopic observations of DAPI-stained material were made to determine the number of nuclei in cells/hyphal compartments at various stages in the life-cycle of the fungus. Our studies revealed that while the basidiospores were consistently unicellular and uninucleate at the time of discharge, the hyphae of $A$. anacardiicola were consistently dikaryotic. Coupled with the observed inability of a single basidiospore to establish a mycelium, our study indicates that dikaryotization is essential for hypha formation and infection of the coccid in the lifecycle of A. anacardiicola.
\end{abstract}

Key words - Fungus-insect-symbiosis - Pucciniomycotina - Septobasidiaceae - scale-insect yeast-stage

\section{Introduction}

Auriculoscypha anacardiicola (Basidiomycota, Pucciniomycotina, Septobasidiaceae) is a fungus currently known only from southwest India where it is seen only on the bark of anacardiaceous trees in obligate association with a coccid (Reid \& Manimohan 1985, Lalitha \& Leelavathy 1990, Lalitha 1992, Kumar et al. 2007; Fig. 1). It is seen invariably associated with a coccid, Neogreenia zeylanica, belonging to the scale-insect family Margarodidae. The fungus gets its nourishment from the coccid using its haustoria that enter the body cavity of the insect and submerge in the haemolymph, while the insect feeds on the sap of the sieve tubes of host trees using its stylet thus involving interactions between three trophic levels. The basidiocarps of the fungus emerge from a tubercle partially immersed in the bark of the host tree within which the enslaved coccids reside. The number of coccids inside a tubercle may vary from one to three. The tubercle enclosing the coccid is composed of both fungal hyphae and host tissues (Lalitha \& Leelavathy 1990, Lalitha 1992).

In several fungi, mating results in a dikaryon, a cell in which two nuclei, one from each parent cell, share a single cytoplasm for a while without undergoing nuclear fusion. This dikaryotic stage is typical in the life-cycles of many fungi primarily in the Basidiomycota (Casselton \& Economou 1985). In several phytopathogenic fungi such as the rusts and the smuts, a prerequisite 
for generating the infectious stage is the mating of two compatible budding haploid cells to generate, after cell fusion, an infective dikaryotic filament. In other words, only a dikaryon can infect the host tissues. After entering the host tissue, the dikaryotic state dominates the period of growth of the fungus occurring during the infectious phase. Similar observations have been made on the basidiomycetous insect pathogen Septobasidium (Couch 1938). There is the possibility of a similar scenario occurring in the life-cycle of A. anacardiicola. The present study wanted to verify the hypothesis that dikaryotization is a prerequisite for hypha formation and coccid-infection in the life-cycle of A. anacardiicola.
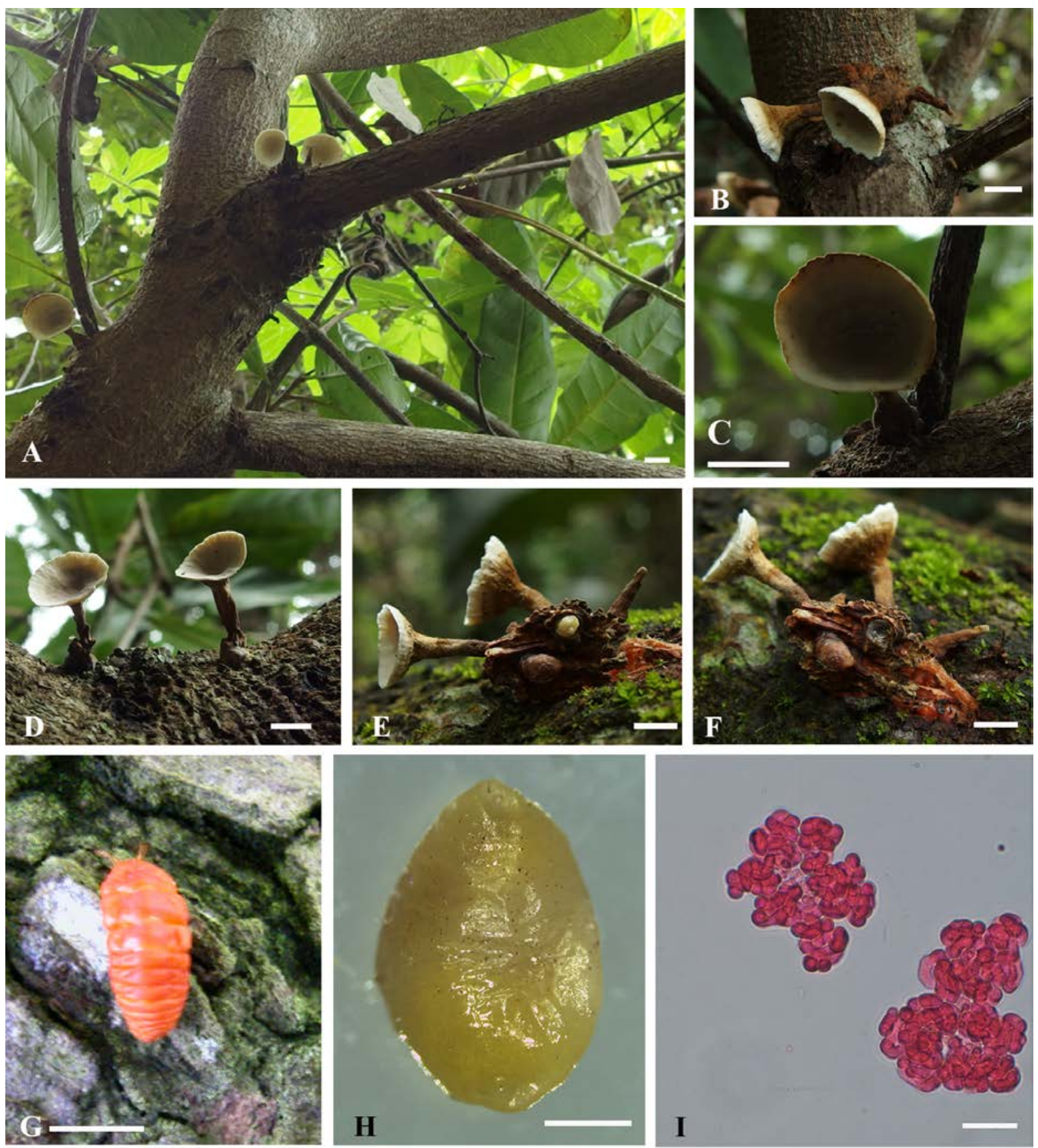

Fig. 1 - The Auriculoscypha anacardiicola symbiosis. A-D Basidiocarps of A. anacardiicola. E Scooped out basidiocarp of $A$. anacardiicola showing the basal tubercle and the enslaved coccid. F Cut-opened basal tubercle showing waxy coating. G Adult female of the coccid Neogreenia zeylanica. $\mathrm{H}$ Immature coccid of $N$. zeylanica scooped out from the tubercle. I Haustorial coils of A. anacardiicola in the haemolymph of the infected coccid. Scale Bars: $A-G=10 \mathrm{~mm}, \mathrm{H}=0.5$ $\mathrm{mm}, \mathrm{I}=20 \mu \mathrm{m}$. 


\section{Materials \& Methods}

\section{Collection of basidiocarps and the associated coccids}

Basidiocarps of $A$. anacardiicola were collected from the Calicut University campus and nearby areas in Malappuram District of Kerala State, India. Fresh basidiocarps were scooped out (along with the basal tubercles containing the coccids) from the bark of the host trees. All the specimens were collected from mango (Mangifera indica L.) and cashew (Anacardium occidentale L.) trees.

\section{Isolation of cultures}

Cultures of $A$. anacardiicola were isolated from spore prints using the method described by Kumar et al. (2011). Tap water agar (TWA) was used as the medium to germinate basidiospores and to initiate cultures. The low nutritional content of TWA reduced the rate of direct contamination from the basidiocarps and the coccids. The freshly collected basidiocarps were brought to the laboratory and thoroughly rinsed with sterilized water and then vortexed in sterilized water to reduce contamination. To get spore print on the surface of the culture medium, mature basidiocarps were glued on to the lid of a Petri plate in such a way that the hymeneal surface was facing the medium. After getting a spore print on the agar surface, further contamination from the fruit body was prevented by removing the fruit body or replacing the lid with another sterile one. The TWA plates with spore deposits were incubated at room temperature and under fluorescent light on a 12 hour on/off cycle till yeast phase and mycelial colony emerged.

\section{Observation of nuclei}

Each developmental stage in the life-cycle of $A$. anacardiicola such as basidiospores, repetitive spores, yeast cells, hyphae from cultured mycelium, hyphae from infected coccids and hyphae of mature basidiocarps were collected separately and stained with nuclear dyes to count the number of nuclei in each cell or hyphal compartment. Both Giemsa and DAPI staining techniques were used to observe the nuclei. Spore prints were obtained from fresh basidiocarps kept inverted on sterile glass-slides within a humid chamber. The basidiospores obtained on these slides were used for nuclear staining. Small agar blocks from culture plates were used to stain hyphae and yeast cells. The coccids inhabiting within the basal tubercle of the basidiocarps were dissected out and haustorial hyphal coils were collected from these infected coccids. The haemolymph oozing out from the coccid while breaking it with a needle was used to get fungal haustoria. A smear of this fluid made on a clean, sterile glass-slide was used for further staining of fungal haustoria to observe the number nuclei in the haustorial compartments. Thin, freehand sections of the basidiocarps were stained to observe the number nuclei in the hyphal compartments of the basidiocarps.

\section{Giemsa staining}

Giemsa staining was carried out to stain and count nuclei in the cells of A. anacardiicola following the protocol described by Carvalho et al. (2002). Tissues were fixed in Carnoy's fixative (ethanol, glacial acetic acid and lactic acid in 6:1:1 by volume) for 25 minutes. The tissues were then washed in distilled water and hydrolyzed with $1 \mathrm{~N} \mathrm{HCl}$ for $15 \mathrm{~min}$ at $25^{\circ} \mathrm{C}$ followed by $10 \mathrm{~min}$ at $60^{\circ} \mathrm{C}$. After hydrolysis, the tissues were washed with distilled water thrice. The washed material was then stained for 1 hour in a solution containing $10 \mathrm{ml}$ phosphate buffer $(\mathrm{pH} 7)$ with 18 drops of Giemsa solution (1.0 g Giemsa and $54.0 \mathrm{ml}$ methanol in $84.0 \mathrm{ml}$ glycerin). The stained specimens were then mounted in phosphate buffer.

\section{DAPI staining}

DAPI (4, 6-Diamidino-2-phenylindole dihydrochloride) was employed to confirm the results of Giemsa staining. The staining protocol followed is that of Raju (1982). A stock solution of DAPI (Roche, USA) was prepared $(1 \mathrm{mg} / \mathrm{ml})$ in distilled water. The specimens were fixed first in 3:1 ethanol: acetic acid for 10 minutes. After fixation, the specimens were washed in distilled water 
and then suspended in the fluorochrome diluted to a final concentration of 0.1 to $0.5 \mu \mathrm{g} / \mathrm{ml}$ for ten minutes at room temperature. The specimens were then washed twice in distilled water and then suspended in 25\% glycerol solution. 25\% glycerol was used for mounting the stained material.

\section{Recording of microscopic structures}

The photomicrographs of all Giemsa-stained structures of $A$. anacardiicola were taken with an Olympus BX43 microscope fitted with an Olympus DP27 camera. Fluorescent micrographs and the juxtaposed bright-field images were taken with a Leica DM6 B microscope fitted with a Leica DFC450 C camera.

\section{Results}

All the observations made on cells/tissues stained by Giemsa protocol were confirmed by DAPI staining (Figs 2, 3 respectively). The basidiospores were consistently unicellular and uninucleate at the time of discharge (Figs 2A, 3A-B). Soon after discharge, they first become multinucleated. This is followed by septa-formation. The basidiospores with multiple septa were usually found to be uninucleate in each segment (Figs 2B, 3C-F). Segments of multiseptate basidiospore with more than one nucleus also were occasionally noticed (Fig. 2D). All of them, however, were in the actively yeast-producing stage. Almost all the yeast cells were uninucleate (Figs 2C, E, 3I-J). Multinucleate yeasts were occasionally noticed (Fig. 2F), which were actively budding. Germinating yeast cells producing one or more germ-tubes were also noticed. In all such cases, the emerging germ tubes were uninucleate (Figs 2I, 3K).

The fusion of germ-tubes arising from yeast cells was observed commonly in the initial stages of culture establishment. In such cases, the compartments of hyphae emerging from the fusion product were observed to be binucleate (Figs 2J, 3N-P). It was these hyphae with binucleate compartments that developed into the mycelium. The compartments of the conidiophores were dikaryotic (Fig. 2H, L). Microconidia that developed from these dikaryotic conidiophores also were binucleate (Fig. 3G-H). Such microconidia were observed to be able to grow into hyphae with each compartment containing two nuclei. Each cell of the haustorial hyphal coils was binucleate (Figs 2G, K, 3L-M). The hyphae from the basidiocarps consistently showed two nuclei in each compartment (Figs 2M, 3Q-R).

\section{Discussion}

Dikaryotization is an essential feature in the life-cycle of rusts, smuts and some allied fungi (Petersen 1974, Littlefield \& Heath 1979, Coelho et al. 2017). A dikaryotic filament developed from the fusion of compatible yeast cells is essential for generating an infectious stage in these fungal groups. Henk \& Vilgalys (2016) demonstrated that individual Septobasidium colonies were composed of a single heterozygous dikaryotic mycelium. The present study showed that all types of hyphae, including those of the basidiocarps, haustorial hyphal coils isolated from the enslaved coccids and the cultured mycelium of A. anacardiicola, were dikaryotic while the basidiospores and secondary blastospores were monokaryotic. These observations indicate that the process of dikaryotization is a crucial step in the life-cycle of $A$. anacardiicola. Our observations indicate that only dikaryotic hyphae can establish infection inside the coccid by forming haustorial hyphal coils, eventually leading to the production of basidiocarps.

Henk \& Vilgalys (2016) observed monokaryotic hyphae of Septobasidium ramorum in the associating infected insects and they inferred that the basidiospores were the infective life stage in S. ramorum. But this observation was specific to S. ramorum. Couch (1938) who first elucidated the complete life-cycle of Septobasidium, had the opinion that the infective stage could be basidiospores or bud cells that formed from the basidiospores and the insects were very rarely or never directly infected by the fungal hyphae. According to Oberwinkler (2017), infection with basidiospores is functionally impossible and the yeasts are the potentially infectious agents in Septobasidiales. Conjugation of compatible yeasts within the insect body leads to the formation of haustorial hyphal coils of distinct shapes and finally, they grow out and form hyphal mats 
(Oberwinkler 2017). The results of the present study indicate that a similar process occurs in $A$. anacardiicola as well.
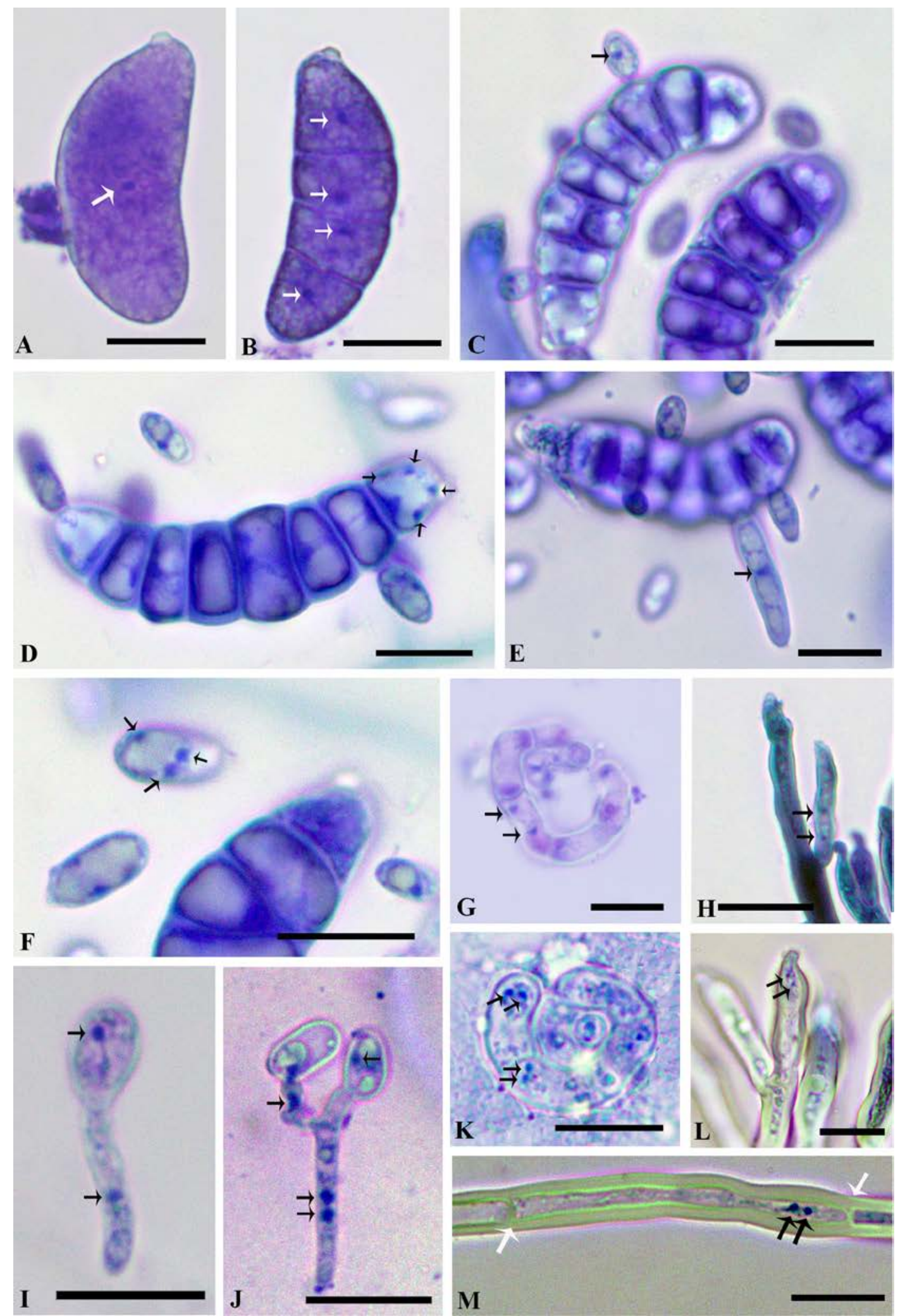

Fig. 2 - Giemsa-stained spores and hyphae of A. anacardiicola. A Aseptate uninucleate basidiospore. B Multiseptate multinucleate basidiospore. C Uninucleate blastospore. D A multinucleate segment of multiseptate basidiospore. E Uninucleate blastospore. F Multinucleate blastospore. G, K Binucleate cells of a haustorial coil. H, L Binucleate conidiophore. I Uninucleate blastospore with uninucleate germ-tube. J fusion between two 
blastospores and formation of a dikaryotic hypha. M Binucleate hyphae from basidiocarp. Scale Bars: A-G, I-M $=10 \mu \mathrm{m}, \mathrm{H}=20 \mu \mathrm{m}$. Arrows indicate nuclei in A-N. In M, black arrows indicate nuclei and white arrows indicate septa.
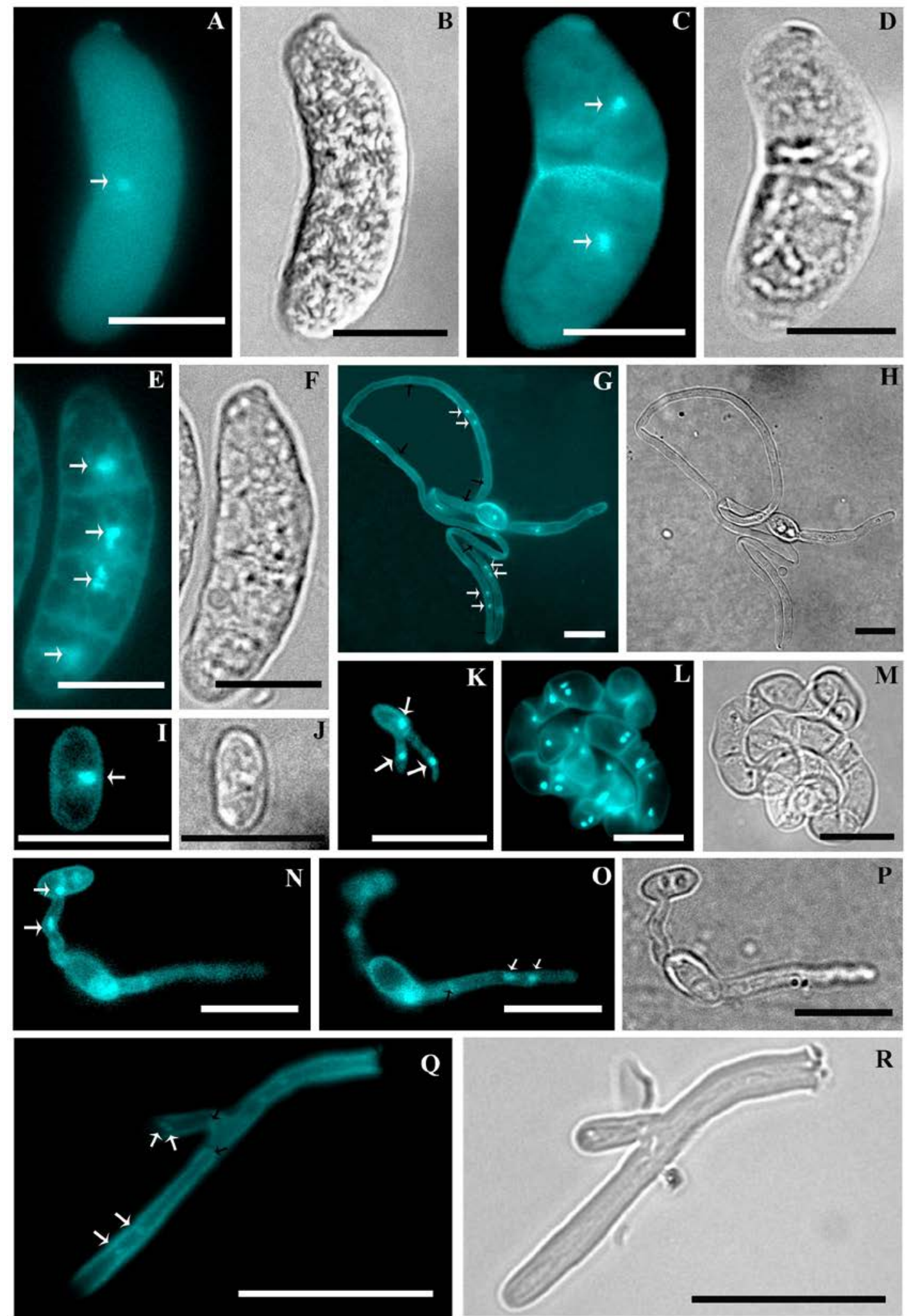

Fig. 3 - DAPI-stained spores and hyphae of A. anacardiicola. A-B Uninucleate basidiospore. C-D Uniseptate basidiospore. E-F Multiseptate basidiospore. G-H Binucleate compartments of hypha emerging from dikaryotic microconidia. I-J Uninucleate blastospore. K Uninucleate germtubes from blastospores. L-M Binucleate cells of haustorial coils. N-P The fusion between 
blastospores. Q-R Binucleate compartments of hypha from basidiocarp. Scale Bars: A-J, L-P = 10 $\mu \mathrm{m}, \mathrm{K}, \mathrm{Q}-\mathrm{R}=25 \mu \mathrm{m}$. White arrows indicate nuclei; black arrows indicate septa.

The observation that all hyphae of $A$. anacardiicola are dikaryotic suggests that the monokaryotic cells must fuse to form the hyphae. This has been further supported by the observation of two blastospores fusing and forming a dikaryotic hypha. Although there is a chance for basidiospores to fall or attach on the insect's body, instead of its direct infection, the blastospores (yeast cells) developing from the basidiospores may be undergoing fusion leading to infection and formation of haustorial hyphal coils. Kumar et al. (2011) have reported the inability of single basidiospores of $A$. anacardiicola to form a mycelium. Mycelial cultures of this fungus were obtained only when spore prints made to fall on agar plates were incubated. The basidiospores in these spore deposits always produced yeast colonies at first which subsequently became mycelial (Kumar et al. 2011). Coupled with this observed inability of a single basidiospore to establish a mycelium, our study indicates that dikaryotization is essential for hypha formation and infection of the coccid in the life-cycle of $A$. anacardiicola.

\section{Acknowledgements}

This work was first presented as a poster at the Asian Mycological Congress held in Tsu, Mie, Japan in October 2019. We are thankful to Dr T. K. Arun Kumar for critical reading of the manuscript.

\section{References}

Carvalho MDF, Baracho MS, Baracho IR. 2002 - An investigation of the nuclei of Hülle cells of Aspergillus nidulans. Genetics and Molecular Biology 25: 485-488.

Casselton LA, Economou A. 1985 - Dikaryon formation. In: Moore D., Casselton L. A., Wood D. A. and Frankland J. C. (Eds.) Developmental biology of higher fungi. Cambridge University Press, pp. 213-229.

Coelho MA, Bakkeren G, Sun S, Hood ME, Giraud T. 2017 - Fungal sex: The Basidiomycota. Microbiology Spectrum 5(3): FUNK-0046-2016.

Couch JN. 1938 - The genus Septobasidium. University of North Carolina Press, Chapel Hill.

Henk DA, Vilgalys R. 2016 - Genetic structure within Septobasidium colonies suggests outcrossing and frequent non-self-fusion. Fungal Genomics \& Biology 6 (2): 1000143.

Kumar TKA, Celio GJ, Matheny PB, McLaughlin DJ et al. 2007 - Phylogenetic relationships of Auriculoscypha based on ultrastructural and molecular studies. Mycological Research 111: 268-74.

Kumar TKA, Jisha KC, Jisha ES, Manimohan P. 2011 - Isolation and in vitro cultivation of Auriculoscypha anacardiicola D.A. Reid et Manim., an insect-associated and potentially medicinal fungus from India. International Journal of Medicinal Mushrooms 13: 273-280.

Lalitha CR. 1992 - Studies on the biology and taxonomy of the genus Auriculoscypha. PhD thesis. The University of Calicut.

Lalitha CR, Leelavathy KM. 1990 - A coccid-association in Auriculoscypha and its taxonomic significance. Mycological Research 94(4): 571.

Littlefield LJ, Heath MC. 1979 - Ultrastructure of Rust Fungi. Academic Press, New York.

Oberwinkler F. 2017 - Yeasts in Pucciniomycotina. Mycological Progress 16: 831-856.

Petersen R. 1974. The rust fungus life-cycle. The Botanical Review 40: 453-513.

Raju NB. 1982 - Easy methods for fluorescent staining of Neurospora nuclei. Fungal Genetics Reports 29(1): 24-25.

Reid DA, Manimohan P. 1985 - Auriculoscypha, a new genus of Auriculariales (Basidiomycetes) from India. Transactions of the British Mycological Society 85: 532-35. 